\title{
Basal Forebrain Cholinergic Neurons Primarily Contribute to Inhibition of Electroencephalogram Delta Activity, Rather Than Inducing Behavioral Wakefulness in Mice
}

\author{
Li Chen', Dou Yin', Tian-Xiao Wang',2, Wei Guo', Hui Dong',2, Qi Xu', Yan-Jia Luo',2, Yoan Cherasse', \\ Michael Lazarus ${ }^{3}$, Zi-long Qiu ${ }^{4}$, Jun Lu ${ }^{5}$, Wei-Min Qu ${ }^{*, 1,2}$ and Zhi-Li Huang ${ }^{*, 1,2}$ \\ 'State Key Laboratory of Medical Neurobiology, Department of Pharmacology and Shanghai Key Laboratory of Bioactive Small Molecules, School \\ of Basic Medical Sciences, Fudan University, Shanghai, China; ${ }^{2}$ The Institutes of Brain Science and Collaborative Innovation Center for Brain Science, \\ Fudan University, Shanghai, China; ${ }^{3}$ International Institute for Integrative Sleep Medicine (WPI-IIIS), University of Tsukuba, Tsukuba, Ibaraki, Japan; \\ ${ }^{4}$ Institute of Neuroscience, Shanghai Institute of Biological Sciences, Chinese Academy of Sciences, Shanghai, China; ${ }^{5}$ Department of Neurology, \\ Division of Sleep Medicine, Harvard Medical School and Beth Israel Deaconess Medical Center, Boston, MA, USA
}

\begin{abstract}
The basal forebrain (BF) cholinergic neurons have long been thought to be involved in behavioral wakefulness and cortical activation. However, owing to the heterogeneity of BF neurons and poor selectivity of traditional methods, the precise role of BF cholinergic neurons in regulating the sleep-wake cycle remains unclear. We investigated the effects of cell-selective manipulation of BF cholinergic neurons on the sleep-wake behavior and electroencephalogram (EEG) power spectrum using the pharmacogenetic technique, the 'designer receptors exclusively activated by designer drugs (DREADD)' approach, and ChAT-IRES-Cre mice. Our results showed that activation of BF cholinergic neurons expressing hM3Dq receptors significantly and lastingly decreased the EEG delta power spectrum, produced low-delta non-rapid eye movement sleep, and slightly increased wakefulness in both light and dark phases, whereas inhibition of BF cholinergic neurons expressing hM4Di receptors significantly increased EEG delta power spectrum and slightly decreased wakefulness. Next, the projections of BF cholinergic neurons were traced by humanized Renilla green fluorescent protein (hrGFP). Abundant and highly dense hrGFP-positive fibers were observed in the secondary motor cortex and cingulate cortex, and sparse hrGFP-positive fibers were observed in the ventrolateral preoptic nucleus, a known sleep-related structure. Finally, we found that activation of BF cholinergic neurons significantly increased c-Fos expression in the secondary motor cortex and cingulate cortex, but decreased c-Fos expression in the ventrolateral preoptic nucleus. Taken together, these findings reveal that the primary function of BF cholinergic neurons is to inhibit EEG delta activity through the activation of cerebral cortex, rather than to induce behavioral wakefulness.

Neuropsychopharmacology (2016) 4I, 2133-2146; doi:10.1038/npp.2016.13; published online 17 February 2016
\end{abstract}

\section{INTRODUCTION}

The basal forebrain $(\mathrm{BF})$ is a collection of subcortical structures, including the substantia innominate (SI), horizontal limb of the diagonal band (HDB), and magnocellular preoptic nucleus (MCPO), that act as the main source of acetylcholine $(\mathrm{ACh})$ released in the brain. Cholinergic neurons in the $\mathrm{BF}$ were thought to play an important role in promoting behavioral wakefulness (Jones, 2005). Microinjection into the $\mathrm{BF}$ of adenosine, a neuromodulator proved to inhibit $\mathrm{BF}$ cholinergic neurons, produced a dramatic decrease in wakefulness by $50 \%$ (Portas et al, 1997).

*Correspondence: Professor W-M Qu or Professor Z-L Huang, Department of Pharmacology, School of Basic Medical Sciences, Fudan University, Shanghai 200032, P. R. China, Tel: +86 21 54237043, Fax: +86 21 54237I03, E-mail: quweimin@fudan.edu.cn or huangzl@fudan.edu.cn

Received 5 September 20 I5; revised 24 November 20 I 5; accepted 22 December 2015; accepted article preview online 22 January 2016
Chemical lesion of $69 \%$ BF cholinergic neurons by 192-IgG-saporin has been shown to increase non-rapid eye movement (non-REM, NREM) sleep by 13\% (Kaur et al, 2008). However, at the same time, several previous results showed that BF cholinergic lesions only changed electroencephalogram (EEG) activity, but did not alter the sleep-wake profile (Blanco-Centurion et al, 2006; Fuller et al, 2011). Berntson et al (2002) reported that lesions of $\mathrm{BF}$ cholinergic neurons attenuated the high-frequency EEG activity, but did not reduce the amount of wakefulness. Taken together, the precise role of $\mathrm{BF}$ cholinergic neurons in regulating sleep-wake behavior has not yet been clarified.

Cholinergic neurons account for only 5\% of the total cell population in the BF (Gritti et al, 2006). The BF contains other transmitter phenotype neurons, including GABAergic and glutamatergic neurons, which differ from cholinergic neurons in firing pattern across the sleep-wake state (Hassani et al, 2009; Semba, 2000; Takahashi et al, 2009). However, the techniques employed by previous 
studies, either pharmacological stimulation or chemical lesion, could not clearly distinguish cholinergic neurons from other neurons. Therefore, previous contradictory results may be due to the heterogeneity of $\mathrm{BF}$ neurons and poor selectivity of traditional approaches.
In present studies, we investigated the exact role of $\mathrm{BF}$ cholinergic neurons in regulating the sleep-wake behavior and EEG power spectrum by a new pharmacogenetic technique, 'designer receptors exclusively activated by designer drugs (DREADD)', which can manipulate the

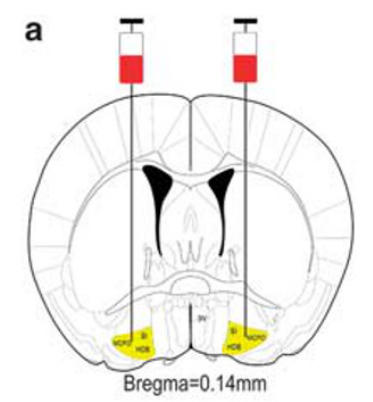

b
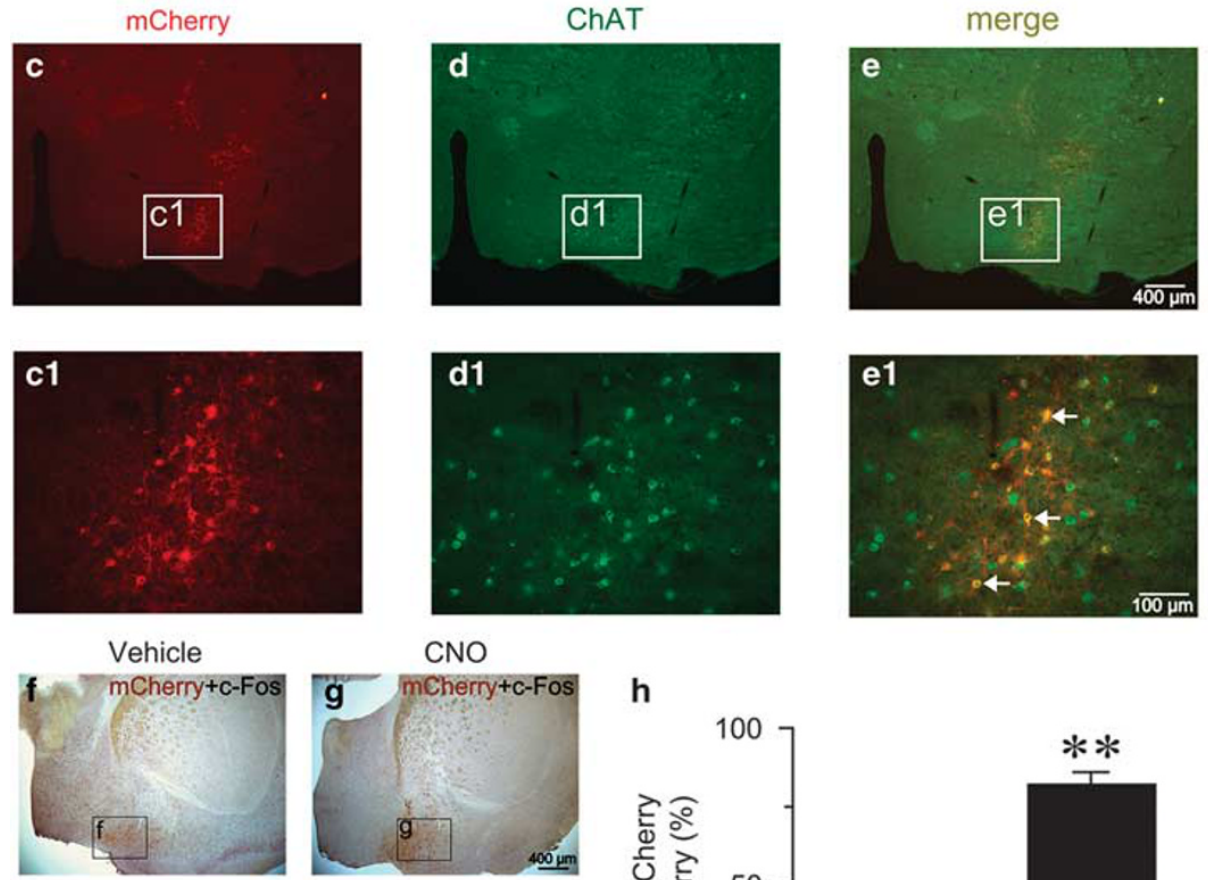

\section{CNO}
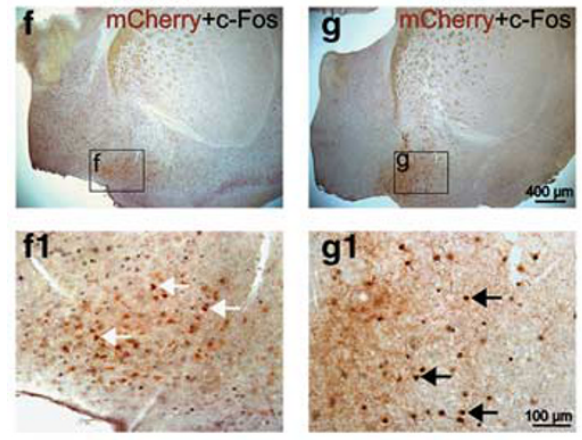

h

i
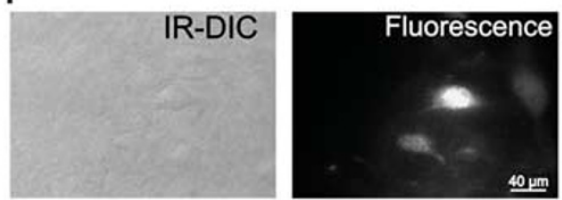

k

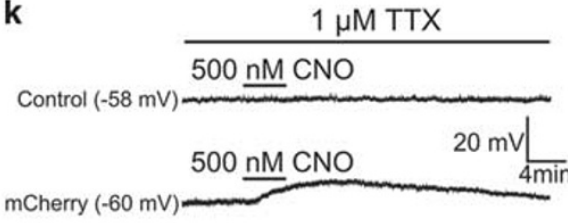

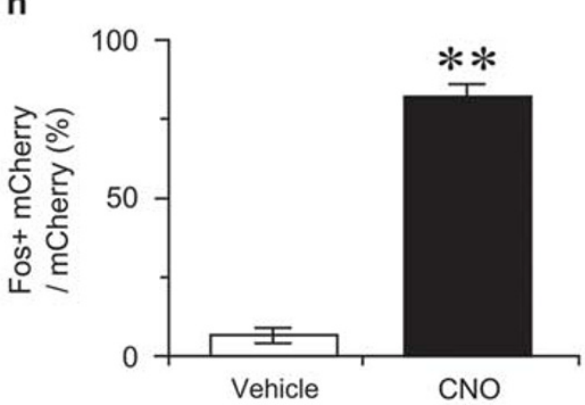

j

500 nM CNO

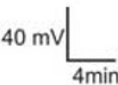

$-57 \mathrm{mV}$

I

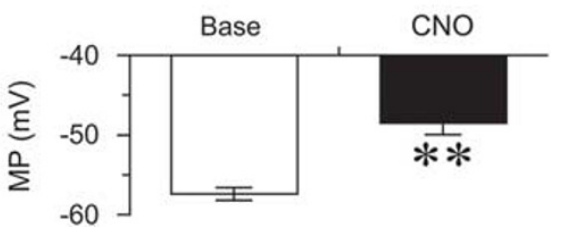


activity of specific neurons based on the principle of Cre/LoxP recombination (Farrell and Roth, 2013). Utilizing ChAT-IRES-Cre transgenic mice in which Cre-recombinase is expressed exclusively in cholinergic neurons (Rossi et al, 2011), we selectively expressed modified G protein-coupled muscarinic receptors (excitatory $\mathrm{hM} 3 \mathrm{Dq}$ receptors and inhibitory hM4Di receptors) in $\mathrm{HDB} / \mathrm{SI} / \mathrm{MCPO}$ cholinergic neurons. Both hM3Dq and hM4Di receptors have lost affinity for endogenous $\mathrm{ACh}$, but can be activated by a synthetic and biologically inert ligand, clozapine- $N$-oxide (CNO). Therefore, using this system, we could manipulate the activity of BF cholinergic neurons cell-specifically and non-invasively. Then we traced the projections of $\mathrm{BF}$ cholinergic neurons using humanized Renilla green fluorescent protein (hrGFP), and examined the effects of activating $\mathrm{BF}$ cholinergic neurons on the $\mathrm{c}$-Fos expression of sleep-wake related structures. Our results clearly reveal that $\mathrm{BF}$ cholinergic neurons mainly contribute to the inhibition of EEG delta activity through the activation of cerebral cortex, rather than to induce behavioral wakefulness.

\section{MATERIALS AND METHODS}

\section{Animals}

Adult male ChAT-IRES-Cre mice (Rossi et al, 2011) and non-Cre-expressing littermate mice (8-12 weeks old, 20-25 g) were used in this study. Mice were bred and housed at an automatically controlled $12 \mathrm{~h}$ light $/ 12 \mathrm{~h}$ dark cycle (lights on at 0700). All procedures were approved by the Medical Experimental Animal Administrative Committee of Basic Medical Sciences School, Fudan University.

\section{Stereotaxic Adeno-Associated Viral (AAV) Injection}

Naive mice were anesthetized with chloral hydrate $(350 \mathrm{mg} /$ $\mathrm{kg}$, i.p.) and then placed in a stereotaxic apparatus. To selectively express the $\mathrm{hM} 3 \mathrm{Dq}$ or $\mathrm{hM} 4 \mathrm{Di}$ receptors in $\mathrm{BF}$ cholinergic neurons, we delivered bilateral microinjection of AAV vectors expressing the hM3Dq or hM4Di receptors in a Cre-dependent configuration (hSyn-DIO-hM3Dq-mCherryAAV or hSyn-DIO-hM4Di-mCherry-AAV) into the BF (coordinates: $\mathrm{AP}=+0.1 \mathrm{~mm}, \mathrm{LR}= \pm 1.3 \mathrm{~mm}, \mathrm{DV}=-4.8 \mathrm{~mm}$ ) (Paxinos and Franklin, 2001). Microinjection of the AAV vector $\left(250 \mathrm{nl} /\right.$ side, $6 \times 10^{12}$ particles $\left./ \mathrm{ml}\right)$ was performed by a compressed air delivery system as described in our previous study (Lazarus et al, 2011).

\section{Polygraphic Recordings and Vigilance State Analysis}

At 14 days after AAV injection, mice were implanted with EEG and electromyogram (EMG) electrodes for polysomnographic recordings. To monitor EEG signals, two stainless steel screws were inserted through the skull of the cortex (AP, $+1.0 \mathrm{~mm}$; LR, $-1.5 \mathrm{~mm}$ from bregma or lambda) and served as EEG electrodes. The EMG activity was monitored by stainless steel Teflon-coated wires bilaterally placed in both trapezius muscles. All electrodes were attached to a microconnector and fixed to the skull with dental cement, as described previously (Qu et al, 2008). Cortical EEG and EMG signals were amplified and filtered and then recorded by using SLEEPSIGN (Kissei Comtec). After EEG/EMG implantation, mice were housed individually in transparent barrels for 10 days, to eliminate the pain and stress of mice from surgery. Then mice were connected with cables and given 4 days for adaptation in an insulated soundproofed recording chamber before EEG/EMG recording.

The results of polygraphic recordings were automatically scored offline in $4 \mathrm{~s}$ epochs as wakefulness, REM sleep, and NREM sleep by SLEEPSIGN according to standard criteria (Huang et al, 2005). To study spontaneous sleep-wakefulness cycles, mice were injected with saline and monitored for $24 \mathrm{~h}$; this period served as a baseline for the second day. In the second day, the same mice were injected of $\mathrm{CNO}$ (Sigma-Aldrich; i.p.) and sleep-wakefulness parameters were recorded.

\section{EEG Power Spectrum Analysis}

In the analysis of the EEG power spectrum, the data obtained during the experimental day were standardized and expressed as percentages of the corresponding time on the baseline day (the day preceding the experimental day). $\mathrm{CNO}$ group mice were injected with vehicle on the baseline day and injected with $\mathrm{CNO}$ (Sigma-Aldrich; i.p.) on the experimental day. The vehicle group mice were injected with vehicle on two consecutive days. The EEG power spectrum was calculated using fast Fourier transform for the frequency range $0-25 \mathrm{~Hz}$, with a resolution of $0.25 \mathrm{~Hz}$ (Qu et al, 2010). To analyze the EEG frequency bands, relative power bins were summed as: delta, $0.5-4 \mathrm{~Hz}$; theta, 6-10 Hz; alpha, 12-14 Hz; and beta, 15-25 Hz.

\footnotetext{
Figure I Selective activation of basal forebrain (BF) cholinergic neurons by designer receptors exclusively activated by designer drugs (DREADD) in vivo and in vitro. (a) Coronal section shows the injection target (highlighted area) in ChAT-IRES-Cre transgenic mice. (b) Schematic representation of DIO-hM3Dq or hM4Di-mCherry-AAV vectors which are under the control of hSyn promoter. ITR, inverted terminal repeat; WPRE, woodchuck hepatitis virus posttranscriptional regulatory element. (c) Microinjection of DIO-hM3Dq-mCherry-AAV into the BF of ChAT-IRES-Cre mice gave rise to the expression of hM3Dq and mCherry (red). (d) Humanized Renilla green fluorescent protein (hrGFP) immunolabeling indicates the location of cholinergic neurons. (e) Overlaid images of mCherry and hrGFP. (cl, dl, el) Higher-power views of boxed region in (c-e), respectively. White arrows indicate selective expression of mCherry and hM3Dq receptors in cholinergic neurons (el). (f) Representative image of mCherry (brown) and c-Fos (black) expression in the BF after injection of vehicle. $(\mathrm{fl})$ Higher-power view of boxed region in $(\mathrm{f})$, showing that most mCherry-expressing neurons did not express $\mathrm{c}$-Fos (white arrows) after injection of vehicle. (g) Representative image of c-Fos in the BF after injection of I mg/kg clozapine-N-oxide (CNO). (gl) Higher-power view of boxed region in (g), showing that c-Fos was expressed in mCherry-expressing neurons after injection of I mg/kg CNO (black arrows). (h) Percentages of double-labeled neurons in $\mathrm{mCherry-expressing} \mathrm{neurons} \mathrm{after} \mathrm{treatment} \mathrm{with} \mathrm{vehicle} \mathrm{or} \mathrm{CNO}$, respectively $(n=6$ or 7$) .{ }^{* *} P<0.0$ I vs vehicle, assessed by the non-paired, twotailed Student's t-test. (i) IR-DIC and mCherry fluorescence image of recorded hM3Dq-mCherry-expressing neuron in BF slice. (j) Bath application of CNO induced firing in hM3Dq-mCherry-expressing neuron in the absence of TTX. (k) CNO produced depolarization in hM3Dq-mCherry-expressing neuron (bottom), but not neighboring control neuron (top), in the presence of TTX. (I) Mean membrane potential (MP) of hM3Dq-mCherry-expressing neurons before and after the application of $500 \mathrm{nM} \mathrm{CNO} \mathrm{(} n=8$ cells from six animals). ${ }^{* *} p<0.0$ I vs baseline, assessed by two-tailed paired Student's $t$-test.
} 


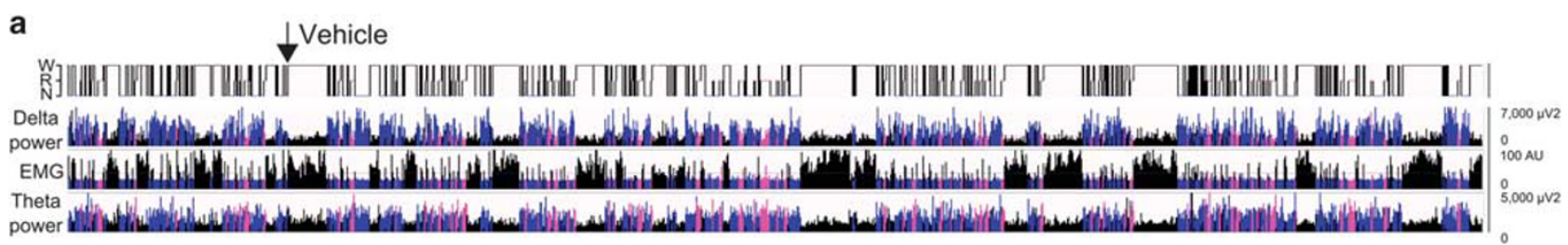

b

b $\quad \perp^{\mathrm{CNO}} 1 \mathrm{mg} / \mathrm{kg}$
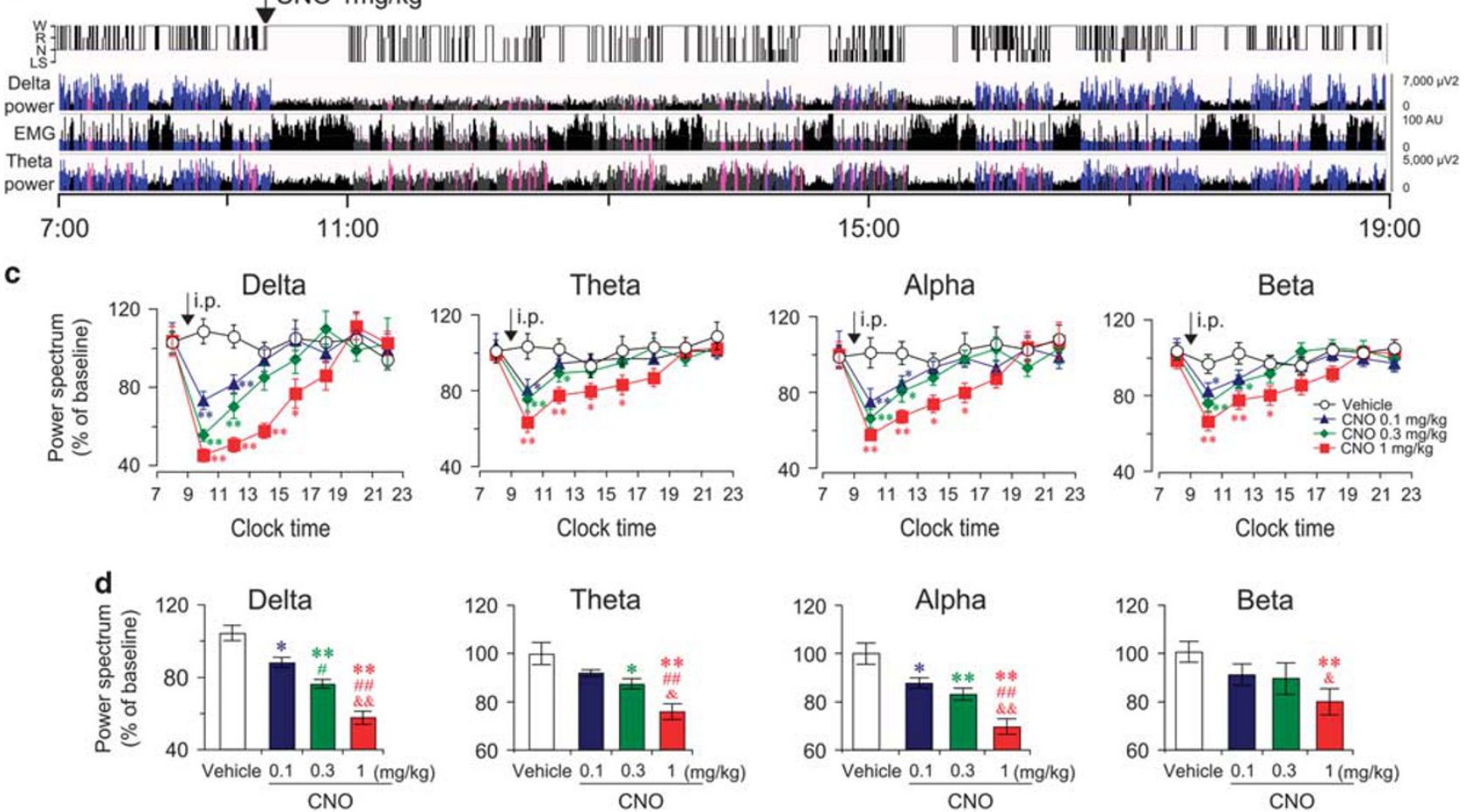

Figure 2 Activation of basal forebrain (BF) cholinergic neurons inhibited electroencephalogram power spectrum. (a, b) Example hypnogram, fast Fourier transform-derived delta and theta power, and electromyogram activity following administration of vehicle (a) or I mg/kg clozapine- $\mathrm{N}$-oxide $(C N O)(b)$ in a ChAT-IRES-Cre mouse with bilateral hM3Dq receptors expression in BF cholinergic neurons. Black $=$ wakefulness $(W)$, pink $=$ REM sleep $(R)$, blue $=$ normal-delta NREM sleep (N), and gray $=\mathrm{CNO}$-induced low-delta state (LS). (c) Time course changes of delta, theta, alpha, and beta power over baseline after injections of different doses of CNO $(n=7-10) .{ }^{*} P<0.05,{ }^{* *} P<0.0$ I vs vehicle, as assessed by repeated-measures ANOVA followed by probable least-squares difference (PLSD) test. (d) Quantitative changes of delta, theta alpha, and beta power over baseline during the $8 \mathrm{~h}$ period after injection (0900-1700, $n=8-10)$. The value of power spectrum after vehicle injection was defined as baseline. Vehicle group and CNO group mice were injected with vehicle and $\mathrm{CNO}$, respectively, on the experimental day. The data were standardized and expressed as percentages of the baseline. ${ }^{*} P<0.05$, ${ }^{* *} P<0.0$ I vs vehicle; ${ }^{\#} P<0.05,{ }^{\# \#} P<0.01$ vs CNO $0.1 \mathrm{mg} / \mathrm{kg}$; ${ }^{\circledR} P<0.05,{ }^{\& \&} P<0.0$ I vs CNO $0.3 \mathrm{mg} / \mathrm{kg}$, assessed by one-way ANOVA followed by the PLSD test.

\section{Immunohistochemistry}

Animals received $\mathrm{CNO}$ or vehicle administration (i.p.) and $2 \mathrm{~h}$ later were deeply anesthetized with chloral hydrate $(350 \mathrm{mg} / \mathrm{kg}$, i.p.), and then fixed by transcardial perfusion with phosphate-buffered saline (PBS) and 4\% paraformaldehyde in PBS. Brains were removed, incubated in $20 \%$ sucrose at $4{ }^{\circ} \mathrm{C}$ until they sank, and then sectioned at $30 \mu \mathrm{m}$ on a freezing microtome. Immunohistochemistry was performed on free-floating sections as previously described (Anaclet et al, 2014).

To detect Fos immunoreactivity, sections were incubated in 3\% hydrogen peroxide for $30 \mathrm{~min}$ at room temperature. The sections were incubated in primary antiserum for $24 \mathrm{~h}$ at $20-23{ }^{\circ} \mathrm{C}$. Primary antiserum included goat anti-c-Fos (1:2000; Santa Cruz Biotechnology) and rabbit anti-mCherry $(1: 10,000$; Clontech). Next sections were incubated in biotinylated secondary antiserum (1:1000; Vector Laboratories) for $2 \mathrm{~h}$. Finally, sections were incubated in avidin-biotin complex (1:1000; Vectastain ABC Elite kit; Vector Laboratories) for $1 \mathrm{~h}$, and incubated in $0.06 \%$ 3,3-diaminobenzidine tetrahydrochloride (DAB) to stain immunoreactive cells for visualization. The sections were stained brown by $\mathrm{DAB}$ or black by $\mathrm{DAB}$ plus $0.05 \%$ cobalt chloride and $0.01 \%$ nickel ammonium sulfate.

The numbers of c-Fos-positive neurons in the ventrolateral preoptic nucleus and tuberomammillary nucleus (TMN) were counted at $+0.02 \mathrm{~mm}$ and $-2.54 \mathrm{~mm}$ from the bregma, respectively. The numbers of c-Fos-positive neurons in the secondary motor cortex and cingulate cortex were counted at $+1.70 \mathrm{~mm}$ from the bregma. The average number of c-Fos immunopositive neurons of the nucleus in each hemisphere was taken to statistical analysis. 


\section{Statistical Analysis}

All data were expressed as mean \pm standard error of the mean. Histograms of the amounts of sleep and wakefulness after vehicle or CNO injection, and membrane potentials of BF cholinergic neurons before and after bath application of CNO, were compared using Student's two-tailed paired $t$-test. The number of c-Fos positive neurons, the number of transition between NREM sleep and wakefulness, and the number and duration of NREM sleep episode were compared using the non-paired, two-tailed Student's $t$-test.

The hourly amounts of each stage for sleep-wake profiles in ChAT-IRES-Cre mice treated with vehicle or CNO were compared using repeated-measures analysis of variance (ANOVA) followed by Fisher's probable least-squares difference (PLSD) test. The EEG power spectra were compared using one-way ANOVA followed by Fisher's PLSD test or the non-paired, two-tailed Student's $t$-test. In all cases, significance was assumed for $P<0.05$.

\section{RESULTS}

\section{Selective Activation of BF Cholinergic Neurons by DREADD}

To selectively activate BF cholinergic neurons by pharmacogenetics, we bilaterally injected AAV vectors containing excitatory hM3Dq receptors (DIO-hM3Dq-mCherry-AAV) into the BF of ChAT-IRES-Cre mice (Figure 1a and b). Robust fluorescence of mCherry was observed in the BF (Figure 1c), and no detectable fluorescence was observed in non-Cre-expressing littermates injected with the same AAV vectors (data not shown). Immunofluorescence staining of brain slices with anti-ChAT antibody revealed that mCherry was expressed exclusively by cholinergic neurons (Figure 1d and e), confirming the requirement for Cre activity to enable the expression of hM3Dq receptors. The effect of hM3Dq receptors in vivo was examined. We administered (i.p.) vehicle and $\mathrm{CNO}(1 \mathrm{mg} / \mathrm{kg})$ into ChAT-IRES-Cre mice expressing hM3Dq receptors at 0900, killed, and fixed them at 1100 . The activity of BF cholinergic neurons was assessed by double staining with anti-c-Fos and anti-ChAT antibodies. Hardly any c-Fos protein was expressed in mCherryexpressing neurons after vehicle injection (Figure 1f). While $\mathrm{CNO}$ injection strongly drove c-Fos protein expression (Figure 1g) in mCherry-expressing neurons, and increased the percentage of double-labeled neurons by $76 \%(P<0.01$; Figure $1 \mathrm{~h}$ ), confirming ligand-induced activation of hM3Dqexpressing BF cholinergic neurons in vivo.

Furthermore, we determined the effect of hM3Dq receptor in vitro using the patch-clamp technique. Fourteen days after DIO-hM3Dq-mCherry-AAV injection, we prepared acute BF slices and performed whole-cell current-clamp recording in mCherry-expressing neurons and neighboring nonmCherry-expressing neurons. The recorded mCherryexpressing neurons (Figure 1i) displayed electrophysiological characteristics similar to those previously reported for cholinergic neurons (Arrigoni et al, 2006), which were mostly silent at resting membrane potentials of about $-57.6 \mathrm{mV}$. Bath application of $\mathrm{CNO}$ drove firing in mCherry-expressing neurons (Figure 1j), and depolarized membrane potentials by $9 \mathrm{mV}$ in the presence of tetrodotoxin (TTX) isolating membrane potential changes from synaptic responses (Figure $1 \mathrm{k}$ and 1 ). Importantly, $\mathrm{CNO}$-induced firing and depolarization were reversible following washout (Figure $1 \mathrm{j}$ and $\mathrm{k}$ ), and $\mathrm{CNO}$ did not affect the firing and membrane potential of neighboring control neurons (non-hM3Dq-expressing) (Figure 1k). These results demonstrated that the DREADD system used in this study can reliably and specifically activate $\mathrm{BF}$ cholinergic neurons.

\section{Pharmacogenetic Activation of BF Cholinergic Neurons Decreases EEG Delta Power Spectrum}

We examined the effects of activating BF cholinergic neurons on EEG power spectrum. Fourteen days after virus injection, either vehicle or $\mathrm{CNO}$ was administered (i.p.) into ChAT-Cre mice at 0900 (light phase) when BF cholinergic activity is weak (Castillo-Ruiz et al, 2010). Following vehicle injection, ChAT-Cre mice displayed a typical light phase hypnogram with substantial amounts of NREM sleep marked by high EEG delta power and low EMG activity (Figure 2a). However, as shown in Figure 2b, after CNO injection, the power of EEG delta spectrum was markedly decreased. We analyzed the EEG power spectrum after injections of $\mathrm{CNO}$ at $0.1,0.3$, and $1.0 \mathrm{mg} / \mathrm{kg}$. The time-course curve (Figure 2c) showed that $\mathrm{CNO}$ at $1.0 \mathrm{mg} / \mathrm{kg}$ significantly decreased the power spectra of delta $(0.5-4 \mathrm{~Hz})$, theta $(6-10 \mathrm{~Hz})$, and alpha $(12-14 \mathrm{~Hz})$ waves for $8 \mathrm{~h}$, and decreased beta $(15-25 \mathrm{~Hz})$ waves for $6 \mathrm{~h}$, as compared with vehicle injection. Both of 0.1 and $0.3 \mathrm{mg} / \mathrm{kg}$ CNO decreased the delta power spectrum for $4 \mathrm{~h}$ (Figure $2 \mathrm{c}$ ). The EEG power spectrum during the $8 \mathrm{~h}$ after CNO injection is summarized in Figure 2d. As shown in Figure 2d, CNO decreased EEG power spectrum in a dose-dependent manner. Injections of $\mathrm{CNO}$ at $0.1,0.3$, and $1.0 \mathrm{mg} / \mathrm{kg}$ decreased delta power spectrum by $16 \%(P<0.05), 28 \% \quad(P<0.01)$, and $47 \%$ $(P<0.01)$, respectively, as compared with vehicle injection. Injections of $\mathrm{CNO}$ at $1.0 \mathrm{mg} / \mathrm{kg}$ decreased theta power spectrum by $24 \%(P<0.01)$, alpha power spectrum by $30 \%$ $(P<0.01)$, and beta power spectrum by $20 \%(P<0.01)$. These results reveal that activation of $\mathrm{BF}$ cholinergic neurons decreased EEG power spectrum, particularly the delta power spectrum. Given that low EEG delta power is indicative of an active cortex (Jones, 2004), these results suggest that BF cholinergic neurons promote the activity of the cerebral cortex.

\section{Activation of BF Cholinergic Neurons Transiently Promotes Wakefulness and Produces Low-Delta NREM Sleep}

Next, we examined the effect of activating BF cholinergic neurons on sleep-wake behavior. We found that after CNO administration, but not after vehicle administration, ChAT-IRES-Cre mice stayed in a state with low delta power (Figure 3a) for long time. In this low-delta state, mice remained motionless with low EMG amplitude, as in the NREM sleep state. Mice could directly enter the REM sleep state in this low-delta state (Figure $3 \mathrm{~b}$ ), as they did from the NREM sleep state. The synchronous video recording revealed that mice kept still, in a typical arched sleep posture with head down and eyes closed, in this low-delta state. Therefore, judged from the EEG, EMG, hypnogram, and 
video recording, the low-delta state after $\mathrm{CNO}$ injection was considered to be NREM sleep (low-delta NREM sleep). We compared the distribution of delta power spectrum of
NREM sleep episode during the $4 \mathrm{~h}$ after vehicle and $1.0 \mathrm{mg} /$ $\mathrm{kg} \mathrm{CNO}$ injection. We found that delta power of most NREM sleep episodes after CNO injection is less than $50 \%$ of

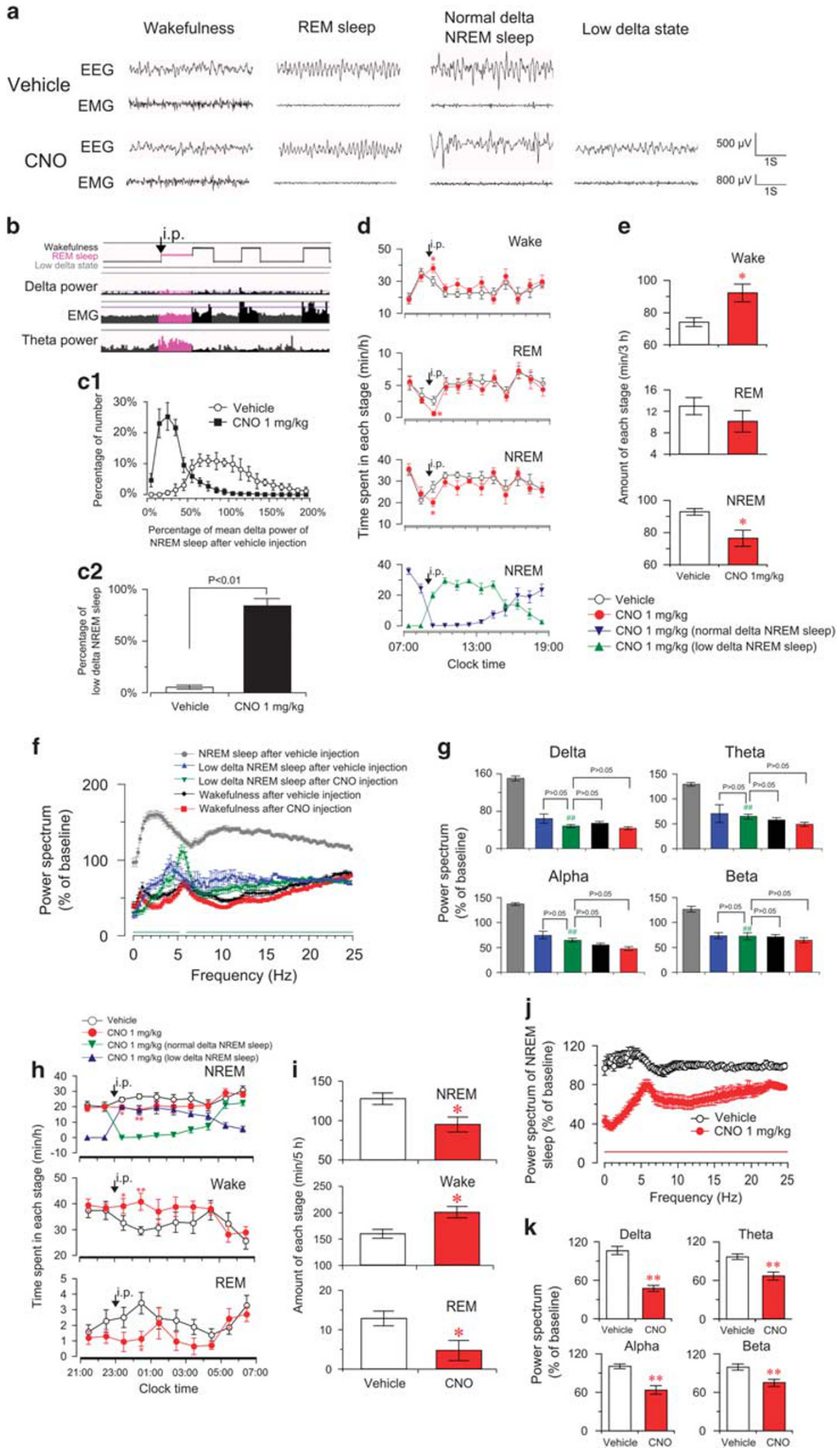


the mean delta power of NREM sleep after vehicle injection (Figure 3c1). According to the analysis of EEG power spectrum, we defined the low delta NREM sleep as the NREM sleep whose delta power is less than $50 \%$ of mean delta power of NREM sleep after vehicle injection. All ChAT-IRES-Cre mice expressing hM3Dq receptors injected with CNO showed substantial low-delta NREM sleep, while during the NREM sleep after vehicle injection, low-delta NREM sleep episodes accounted for only $5 \%$ of total NREM sleep (Figure 3c2). Normal waking and REM sleep states were also observed after $\mathrm{CNO}$ administration, which are similar to that after vehicle administration (Figure 3a). In a word, our results showed that activation of $\mathrm{BF}$ cholinergic neurons induced the low-delta NREM sleep with virtual absence of slow-wave activity.

To better determine the change on sleep-wake behavior, we further classified NREM sleep into normal-delta NREM sleep and low-delta NREM sleep. The time course of sleep-wake profiles showed that CNO injection significantly and lastly decreased normal-delta NREM sleep, with the appearance of substantial low-delta NREM sleep (Figure 3d, bottom). NREM sleep (with normal and low-delta power) significantly decreased during the first hour after CNO injection $(P<0.05$; Figure $3 \mathrm{~d})$, as compared with vehicle injection. Accordingly, wakefulness significantly increased for $1 \mathrm{~h}(P<0.05)$ and exhibited an increased tendency for an additional $2 \mathrm{~h}$. Figure $3 \mathrm{e}$ summarizes the total time that mice spent in wakefulness, REM sleep, and NREM sleep during the $3 \mathrm{~h}$ following CNO administration. The administration of CNO decreased NREM sleep by 17 min $(P<0.05)$ with an increase in wakefulness $(P<0.05)$. At the same time, we found CNO injections increased short NREM sleep episodes, decreased long NREM episodes, shortened the mean duration of NREM sleep episode, and increased the transition from NREM sleep to wake state (Supplementary Figure S1), indicating that activation of $\mathrm{BF}$ cholinergic neurons leads to sleep fragmentation. In addition, we examined the effect of activation of BF cholinergic neurons on locomotor activities. The profiles of locomotor activities of ChAT-Cre mice were not significantly changed after CNO injection in the light phase (Supplementary Figure S2a), consistent with a mild increase in wakefulness. These results indicate that activation of $\mathrm{BF}$ cholinergic neurons transiently increased wakefulness, induced sleep fragmentation, and produced low-delta NREM sleep.

To better understand the nature of low-delta NREM sleep, we analyzed the EEG power spectrum of each state after vehicle or CNO injection. As shown in Figure 3f, the EEG power spectrum of low-delta NREM sleep after CNO injection was decreased in the low-frequency band $(0-5.25 \mathrm{~Hz})$ and higher frequency band $(6-25 \mathrm{~Hz})$ $(P<0.05)$, as compared with NREM sleep after vehicle injection. We compared the power of the delta, theta, alpha, and beta band of each state (Figure 3g). The delta power of CNO-induced low-delta NREM sleep was markedly decreased by $102 \%$ of baseline as compared with NREM sleep after vehicle injection (Figure 3g), but is not statistically different from that of wake state after vehicle or CNO injection (Figure 3g). Given that EEG delta power is indicative of cortical activation (Jones, 2004), these results suggested that, during low-delta NREM sleep after activating $\mathrm{BF}$ cholinergic neurons, the cerebral cortex is activated, even if animals are behaviorally sleep.

\section{Activation of BF Cholinergic Neurons Decreases Delta Power of NREM Sleep}

To determine the effect of enhancing endogenous activity of BF cholinergic neurons on NREM sleep. We administered $\mathrm{CNO} 1 \mathrm{mg} / \mathrm{kg}$ in the dark phase when BF cholinergic neurons are active (Castillo-Ruiz et al, 2010). The time course of sleep-wake profiles showed that $\mathrm{CNO}$ injection at 2300 induced a more obvious increase in wakefulness than injection in the light phase (Figure $3 \mathrm{~h}$ ). After CNO injection, average hourly wakefulness significantly increased for $2 \mathrm{~h}$ and exhibited an increased tendency for an additional $3 \mathrm{~h}$, as

Figure 3 Activation of basal forebrain (BF) cholinergic neurons slightly promoted wakefulness, produced low-delta non-rapid eye movement (NREM) sleep, and decreased electroencephalogram (EEG) power spectrum of NREM sleep. (a) Raw EEG and electromyogram traces of ChAT-IRES-Cre mice with bilateral hM3Dq receptor expression in BF cholinergic neurons following vehicle (top) and clozapine-N-oxide (CNO) (bottom) injection. Note the occurrence of lowdelta state with low-delta and EEG activity after CNO injection. (b) Hypnogram shows that ChAT-IRES-Cre mouse directly entered REM sleep from CNOinduced low-delta state (black arrows). (cl) The distribution of delta power spectrum of NREM sleep episode during the first $4 \mathrm{~h}$ after the injection of vehicle and $1.0 \mathrm{mg} / \mathrm{kg}$ CNO (900 to 1300). Data were standardized and expressed as the percentage of the mean delta power of NREM sleep after vehicle injection of each mouse $(n=6)$. (c2) The percentage of low delta NREM sleep episode in total NREM sleep episode after the injection of vehicle and I.0 mg/kg CNO $(n=6)$, assessed by the non-paired, two-tailed Student's t-test. (d) Time course of vigilance states following vehicle and I mg/kg CNO injections in ChATIRES-Cre mice ( $n=8$ or 9). Horizontal open bars on $x$-axes indicate $12 \mathrm{~h}$ light periods. Note the presence of low-delta NREM sleep and significant decrease of normal-delta NREM sleep after CNO injections (bottom in d). ${ }^{*} P<0.05$ vs vehicle, assessed by repeated-measures ANOVA followed by the probable leastsquares difference (PLSD) test. (e) Total amounts of vigilance states during the first $3 \mathrm{~h}$ after the injection $(900$ to I200) ( $n=8$ or 9$)$. * $P<0.05$ vs vehicle, assessed by two-tailed paired Student's t-test. The comparison of EEG power spectrum of each state after vehicle or $1.0 \mathrm{mg} / \mathrm{kg} \mathrm{CNO}$ injection (f, g) and the EEG power spectrum (including all states) after vehicle injection was selected as baseline. (f) EEG power spectrum changes over baseline during the $8 \mathrm{~h}$ after vehicle or I mg/kg CNO injection ( $n=7-9)$. Horizontal bars indicate statistical difference between NREM sleep after vehicle injection and low-delta NREM sleep after CNO injection $(P<0.05)$, assessed by the non-paired, two-tailed Student's t-test. (g) Quantitative analysis of delta, theta, alpha, and beta power over baseline $(n=7-9),{ }^{\#} P<0.01$ vs NREM sleep after vehicle injection, assessed by one-way ANOVA followed by the PLSD test. Activation of BF cholinergic neurons increased wakefulness and decreased EEG power spectrum of NREM sleep in the dark phase (h-k). (h), Time course of vigilance states after injection of vehicle or $1 \mathrm{mg} / \mathrm{kg}$ CNO at 2300 in mice expressing hM3Dq receptors $(n=8$ or 9). Horizontal filled bars on $x$-axes indicate dark periods. ${ }^{*} P<0.05,{ }^{* *} P<0.01$ vs vehicle, assessed by repeated-measures ANOVA followed by the PLSD test. (i) Total amounts of vigilance states for $5 \mathrm{~h}$ after injection (2300 to $0400, n=8$ or 9). ${ }^{*} P<0.05$ vs vehicle, assessed by two-tailed paired Student's t-test. (j) EEG power spectrum of NREM sleep changes over baseline during $8 \mathrm{~h}$ after CNO injection ( 2300 to $0700, n=8)$. Horizontal bar indicates statistical difference $(P<0.05)$, assessed by the non-paired, two-tailed Student's t-test. ( $k$ ) Quantitative changes in the delta, theta, alpha, and beta power of NREM sleep epochs $(n=8) .{ }^{* *} P<0.0$ I vs vehicle, assessed by the non-paired, twotailed Student's t-test. 
compared with vehicle injection. Accordingly, NREM sleep (with normal and low-delta power) was decreased during the first and second hour by $5 \mathrm{~min}(P<0.05)$ and
10 min $(P<0.01)$, respectively (Figure $3 \mathrm{~h})$. The total time spent in NREM sleep, REM sleep, and wakefulness during the $5 \mathrm{~h}$ after $\mathrm{CNO}$ injection is summarized in
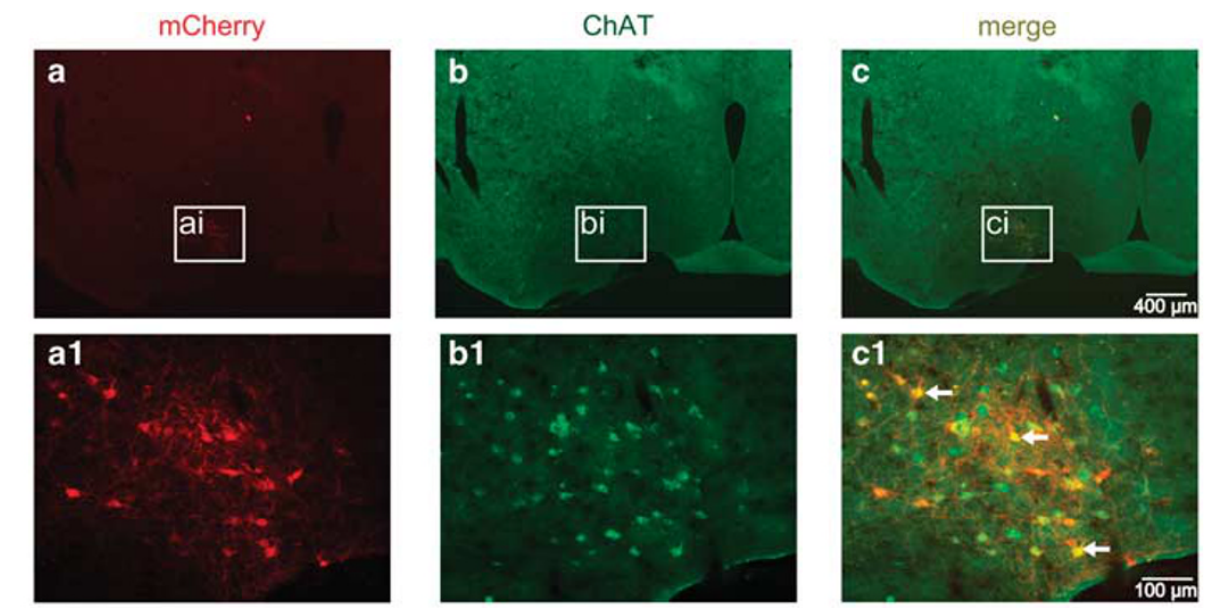

d

$1 \mu \mathrm{M}$ TTX
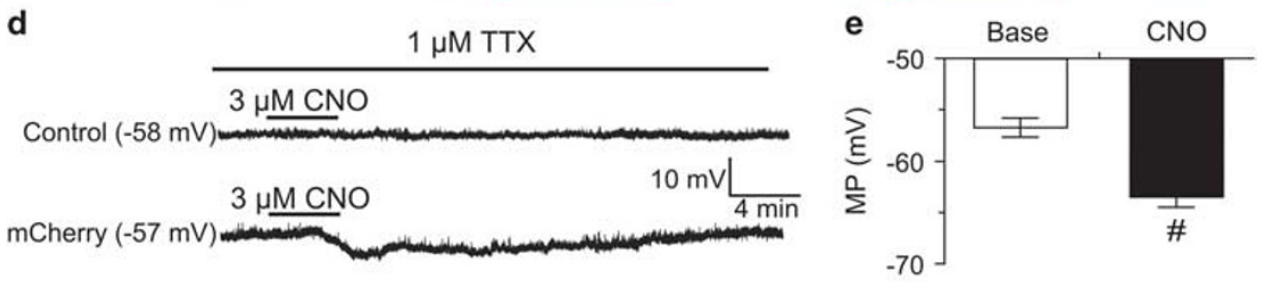

f

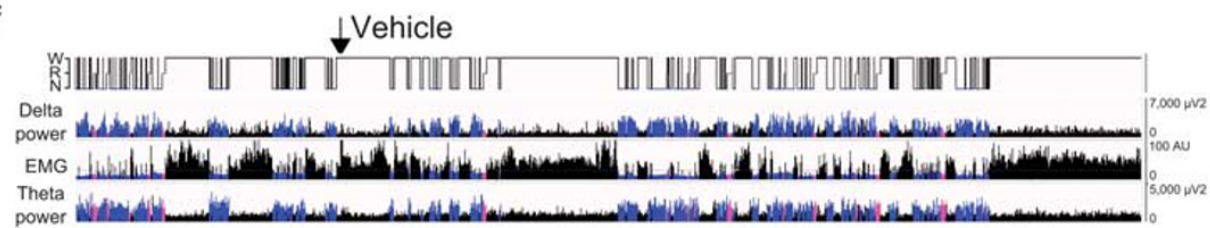

\section{g}

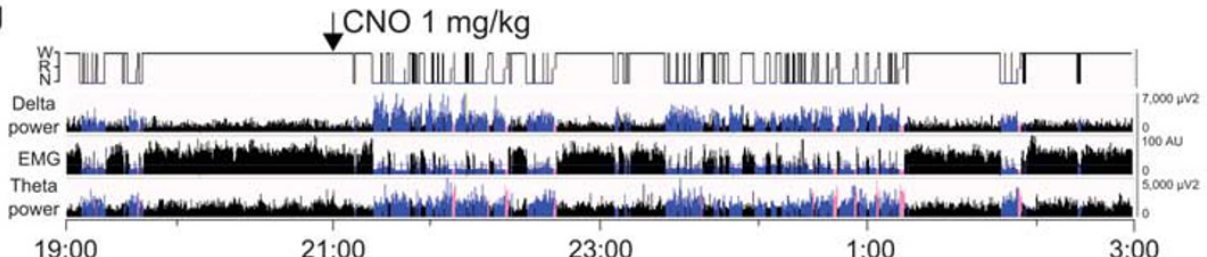

h

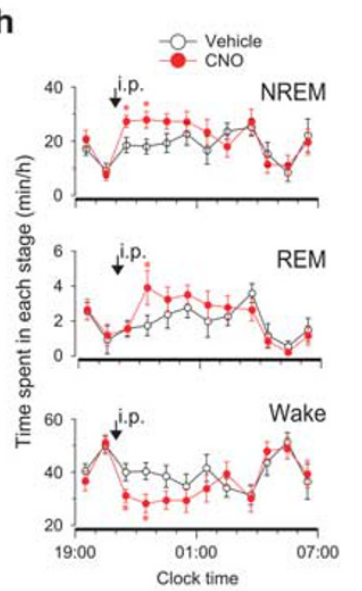

i

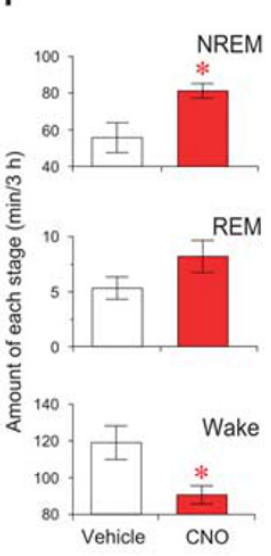

3:00
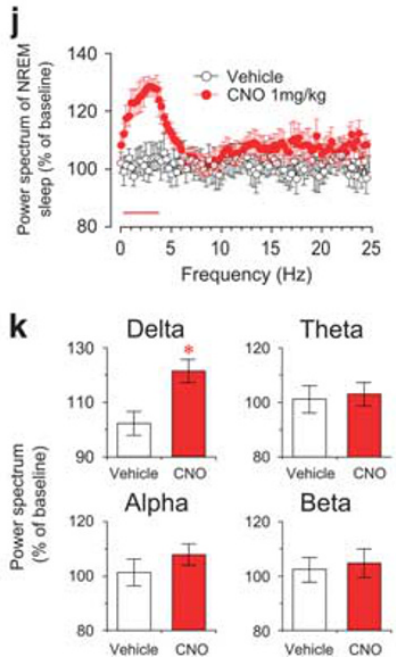
Figure 3i. Injection of $\mathrm{CNO}$ increased wakefulness by 41 min $(P<0.05)$, and decreased NREM sleep by 33 min $(P<0.05)$ (Figure 3i). As shown in Supplementary Figure S2b, activation of BF cholinergic neurons in the dark phase did not increase locomotor activities of mice, as it did in the light phase.

We analyzed the EEG power spectrum of NREM sleep (with normal and low-delta power) during the $8 \mathrm{~h}$ after $\mathrm{CNO}$ injection. As shown in Figure 3j, CNO injection notably decreased the EEG power spectrum of NREM sleep over the frequency range $0-25 \mathrm{~Hz}(P<0.05)$. The delta, theta, alpha, and beta power of NREM sleep were decreased by $59 \%$ $(P<0.01), \quad 30 \% \quad(P<0.01), \quad 37 \% \quad(P<0.01), \quad$ and $\quad 24 \%$ $(P<0.01)$, respectively (Figure $3 \mathrm{k})$. Given that the EEG delta power of NREM sleep is often used as an indicator of need for sleep and sleep depth, which is enhanced after sleep deprivation (Davis et al, 2011), our results indicate that the activation of BF cholinergic neurons decreases the depth of sleep.

\section{Selective Inhibition of BF Cholinergic Neurons Increases Delta Power of NREM Sleep and Decreases Wakefulness}

To test the effects of selectively inhibiting BF cholinergic neurons, we bilaterally injected AAV vectors containing inhibitory hM4Di receptors (DIO-hM4Di-mCherry-AAV) into ChAT-IRES-Cre mice. Robust expression of mCherry was observed in the BF (Figure 4a), and immunofluorescence staining showed that mCherry was specifically expressed on the cholinergic neurons (Figure $4 \mathrm{~b}$ and $\mathrm{c}$ ). The effects of hM4Di receptor on $\mathrm{BF}$ cholinergic neurons were examined in vitro. The membrane potentials of mCherry-expressing neurons, but not non-mCherry-expressing neurons, were hyperpolarized by $7 \mathrm{mV}(P<0.05)$ after bathing $\mathrm{CNO}$ at $3 \mu \mathrm{M}$, and were reversed after washout (Figure $4 \mathrm{~d}$ and e). These results demonstrate that the activity of BF cholinergic neurons can, specifically and effectively, be inhibited by hM4Di receptors.

To determine the effect of attenuating endogenous activity of BF cholinergic neurons on NREM sleep, vehicle or CNO at $1 \mathrm{mg} / \mathrm{kg}$ was injected at 2100 (dark phase) when the activity of BF cholinergic neurons is high. As shown in Figure $4 \mathrm{f}$ and $\mathrm{g}, \mathrm{CNO}$ decreased wakefulness and increased delta power spectrum. The time course of sleep-wake profiles showed that average hourly wakefulness decreased for $2 \mathrm{~h}(P<0.05)$ and exhibited a decreased tendency for additional $1 \mathrm{~h}$, as compared with vehicle injection. Accordingly, NREM sleep increased by $9 \mathrm{~min}(P<0.05)$ and $10 \mathrm{~min}$ $(P<0.05)$ during the first and second hour after $\mathrm{CNO}$ administration, respectively (Figure $4 \mathrm{~h}$ ). The total time spent in NREM sleep, REM sleep, and wakefulness during the $3 \mathrm{~h}$ after CNO injection is summarized in Figure 4i. Injection of CNO significantly increased NREM sleep by $26 \min (P<0.05)$ with a decrease of wakefulness (Figure 4i). Furthermore, we found that CNO injection increased long NREM episode and prolonged mean duration of NREM sleep episode (Supplementary Figure S3), indicating that inhibition of BF cholinergic neurons increases sleep continuity.

To investigate the effect of inhibiting BF cholinergic neurons on NREM sleep, we analyzed the EEG power spectrum of NREM sleep during the $3 \mathrm{~h}$ after CNO injection. CNO injection significantly increased the EEG power of NREM sleep in the frequency range $0.25-3.75 \mathrm{~Hz}(P<0.05)$, as compared with vehicle injection (Figure $4 \mathrm{j})$. Injection of CNO significantly increased the delta power spectrum of NREM sleep by $22 \%(P<0.05)$, but did not significantly change the power of theta, alpha, or beta spectrum (Figure 4k). These results show that inhibition of $\mathrm{BF}$ cholinergic neurons induced NREM sleep, increased EEG delta power, and decreased the activity of the cerebral cortex.

\section{BF Cholinergic Neurons Densely Project toward Cerebral Cortex and Drive Cortical c-Fos Expression}

To explore the underlying mechanism of sleep-wake regulation by $\mathrm{BF}$ cholinergic neurons, we mapped the projections of these neurons. We utilized Cre-dependent AAV vectors encoding hrGFP as an anterograde tracer for long axonal pathways. Twelve days after the unilateral BF injection of DIO-hrGFP-AAV, immunohistochemical assessment confirmed that hrGFP-positive somas were restricted to cholinergic neurons of HDB/SI/MCPO (Figure 5a-d). The hrGFP-positive axons of BF cholinergic neurons were distributed in the hemisphere ipsilateral to the injection site. As shown in Figure 5e and $\mathrm{f}$, we found abundant and high dense hrGFP-positive fibers in the cerebral cortex, particularly in the secondary motor cortex (M2) and cingulate cortex $(\mathrm{Cg})$. At the same time, sparse hrGFP-positive fibers were observed in the VLPO, a known sleep-related structure (Figure $5 \mathrm{~g}$ and $\mathrm{h}$ ). No hrGFP fluorescence was found in the

\footnotetext{
Figure 4 Selective inhibition of basal forebrain (BF) cholinergic neurons by hM4Di receptors decreased wakefulness and increased electroencephalogram (EEG) power spectrum of non-rapid eye movement (NREM) sleep. (a-c) Cre-dependent expression of the hM4Di receptors in BF cholinergic neurons. (a) Microinjection of DIO-hM4Di-mCherry-AAV into the BF of ChAT-IRES-Cre mice gave rise to the expression of mCherry and hM4Di (red). (b) GFP immunolabeling indicates location of cholinergic neurons. (c) Overlaid images of mCherry and GFP. (al, bl, cl) Higher-power views of boxed region in (a-c), respectively. White arrows indicate selective expression of $\mathrm{mCherry}$ and $\mathrm{hM}$ 4Di receptors in BF cholinergic neurons ( $\mathrm{cl}$ ). (d) Bath application of clozapine- $\mathrm{N}$ oxide $(\mathrm{CNO})(3 \mu \mathrm{M})$ resulted in hyperpolarization of BF hM4Di-mCherry-expressing neurons (bottom), but not neighboring control neurons (top), in the presence of TTX in brain slices. (e) Mean resting membrane potentials of hM4Di-mCherry-expressing neurons before and after application of $3 \mu \mathrm{M}$ CNO ( $n=7$ cells from five animals). ${ }^{\#} P<0.05$ vs base membrane potential (MP), assessed by two-tailed paired Student's t-test. (f, g) Example hypnogram, fast Fourier transform-derived delta and theta power, and electromyogram activity following vehicle (f) or I mg/kg CNO (g) administration in mice expressing hM4Di receptors. Black = wakefulness $(W)$, pink $=$ REM sleep $(R)$, blue $=$ NREM sleep $(N)$. (h) Time course of vigilance states following injection of vehicle or $C N O$ ( $n=8$ or 9 ). Horizontal filled bars on $x$-axes indicate dark periods. ${ }^{*} P<0.05$ vs vehicle, assessed by repeated-measures ANOVA followed by the PLSD test. (i) Total amounts of vigilance states during the $3 \mathrm{~h}$ post-injection $(2100$ to 0000$)(n=8$ or 9$)$. ${ }^{*} P<0.05$ vs vehicle, assessed by two-tailed paired Student's $t$-test. (j) EEG power spectrum of NREM sleep changes over baseline during $3 \mathrm{~h}$ after injection of CNO $(n=7$ or 8$)$. Horizontal bar indicates statistical difference $(P<0.05)$, assessed by the non-paired, two-tailed Student's t-test. $(k)$ Quantitative changes in the delta, theta, alpha, and beta power of NREM sleep epochs ( $n=7$ or 8$) .{ }^{*} P<0.05$ vs vehicle, assessed by the non-paired, two-tailed Student's $t$-test.
} 

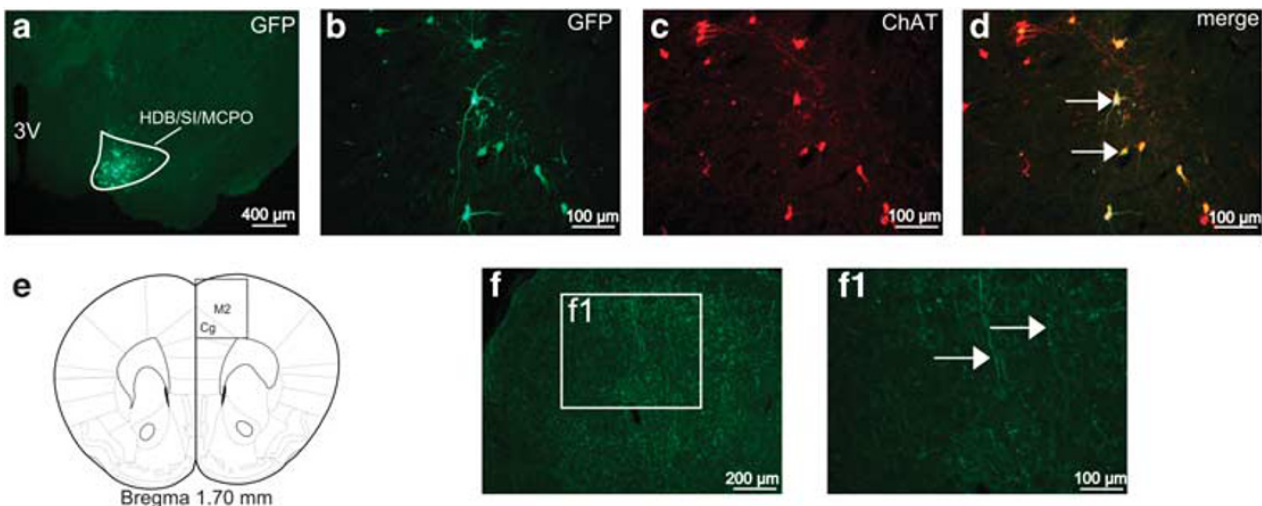

g

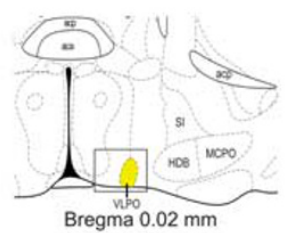

Vehicle
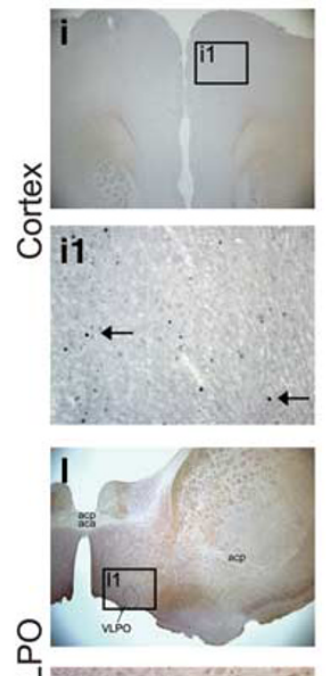

11 h

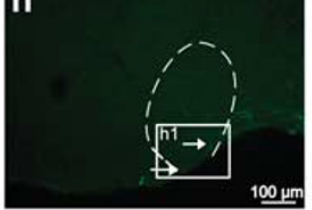

$\mathrm{CNO}$
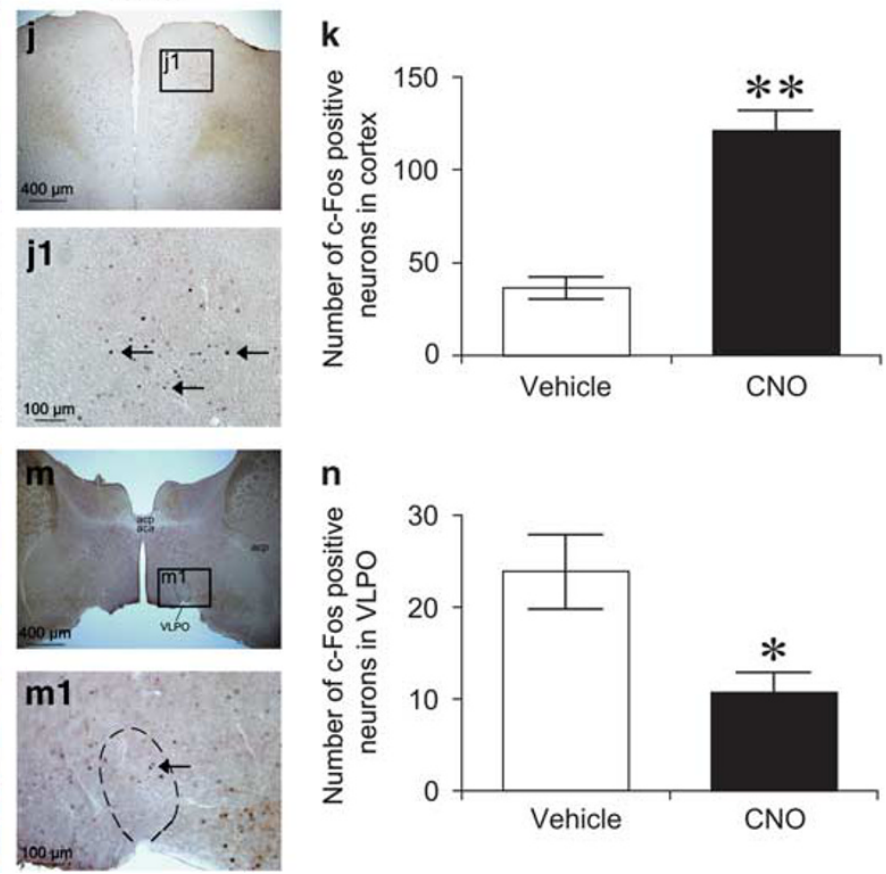

Figure 5 The projection of basal forebrain (BF) cholinergic neurons and the c-Fos expression after activating BF cholinergic neurons by hM3Dq receptors. Cre-dependent expression of humanized Renilla green fluorescent protein (hrGFP) in BF cholinergic neurons (a-d). (a) Coronal section showing unilateral injection target of DIO-hrGFP-AAV (HDB/SI/MCPO, delimited area). (b) Microinjection of DIO-hrGFP-AAV into ChAT-IRES-Cre mice resulted in the expression of hrGFP in the BF. (c) mCherry immunolabeling indicates location of cholinergic neurons in the BF. (d) Overlaid images of hrGFP and mCherry indicate selective expression of hrGFP in BF cholinergic soma (white arrows). (e) Coronal section shows the projection site in cortex from BF cholinergic neurons. Square in (e) delimits the cortical area shown in (f). (f) Microinjection of DIO-hrGFP-AAV into the BF resulted in highly dense expression of hrGFP in the ipsilateral secondary motor cortex (M2) and cingulate cortex (Cg). (g) Schematics show the ventrolateral preoptic nucleus (VLPO, high light area). (h) Microinjection of DIO-hrGFP-AAV into the BF resulted in sparse expression of hrGFP in VLPO. $(\mathrm{fl}, \mathrm{hl})$ Higher power view of boxed region in (f, $h$ ); white arrows indicate projecting fibers from BF cholinergic neurons. acp, anterior commissure posterior, aca, anterior commissure anterior. Representative photomicrographs of c-Fos expression in M2/Cg (i, j) and VLPO $(\mathrm{l}, \mathrm{m})$ of ChAT-IRES-Cre mice expressing hM3Dq receptors after administration of vehicle or I mg/kg clozapine-N-oxide (CNO). (il, jl, II, ml) Higher power view of boxed region in (i, j, l, m), respectively; black arrows indicates the c-Fos positive neurons. Mean numbers of c-Fos-positive neurons in $\mathrm{M} 2 / \mathrm{Cg}(\mathrm{k})$ and $\mathrm{VLPO}(\mathrm{n})$ after activating of BF cholinergic neurons by $\mathrm{CNO}$ treatment $(n=8)$. ${ }^{*} P<0.05$, ${ }^{* *} P<0.01$ vs vehicle injection, assessed by the non-paired, two-tailed Student's t-test.

TMN (data not shown), an important arousal-related structure. Next, we determined the effects of activating BF cholinergic neurons with hM3Dq receptors on the activity of $\mathrm{M} 2 / \mathrm{Cg}$, VLPO, and TMN neurons by analyzing the expression of c-Fos protein. ChAT-IRES-Cre mice were injected with vehicle or CNO $(1 \mathrm{mg} / \mathrm{kg})$ at 0900, killed, and fixed at 1100 . We found that CNO injection significantly increased the number of c-Fos-positive neurons in $\mathrm{M} 2 / \mathrm{Cg}$ by 
233\% relative to vehicle injection $(P<0.01$; Figure $5 \mathrm{i}-\mathrm{k})$, and decreased $c$-Fos expression in the VLPO by 55\% $(P<0.05$; Figures 5l-n), but did not significantly alter c-Fos expression in the TMN (data not shown). Given that c-Fos expression is a biological marker of neural activation, these results suggest that activation of BF cholinergic neurons promotes cortical activity by their innervations to $\mathrm{M} 2 / \mathrm{Cg}$ and other sleep-wake related structures.

\section{DISCUSSION}

Our results show that cell-specific activation of BF cholinergic neurons by DREADD markedly decreased EEG delta power, increased cortical c-Fos expression, and slightly promoted behavioral wakefulness, while inhibition of $\mathrm{BF}$ cholinergic neurons increased EEG delta power and decreased wakefulness, indicating that $\mathrm{BF}$ cholinergic neurons mainly contribute to the inhibition of EEG delta activity through the activation of cerebral cortex, rather than inducing behavioral wakefulness.

Previous pharmacological studies suggest that BF cholinergic neurons play an important role in wakefulness. Microinjections of $\alpha$-amino-3-hydroxy-5-methyl-4-isoxazolepropionic acid (AMPA) and $N$-methyl D-aspartate (NMDA) into the $\mathrm{BF}$, to stimulate $\mathrm{BF}$ cholinergic neurons, produced an increase in wakefulness by about 50\% (Cape and Jones, 2000a; Manfridi et al, 1999). Administration to the BF with neurotensin, a neuropeptide that potently activates cholinergic neurons in vitro, significantly increase waking time (Cape et al, 2000b). However, recent studies in which BF cholinergic neurons were ablated with 192-IgGsaporin have failed to detect significant changes in the sleepwake cycle (Blanco-Centurion et al, 2007; Fuller et al, 2011).

It is reasonable to speculate that inconsistent results could be due to the low cell-selectivity of pharmacological stimulation and chemical ablation, which could not distinguish cholinergic neurons in the $\mathrm{BF}$. As well as cholinergic neurons account for only $5 \%$ of the total number of $\mathrm{BF}$ neurons, the BF also contains GABAergic and glutamatergic neurons, which account for about 35 and 55\%, respectively (Gritti et al, 2006).

Microinjection of AMPA and NMDA into the BF induced the expression of c-Fos protein in cholinergic and GABAergic neurons (Cape and Jones, 2000a), revealing that glutamatergic stimulation excited cholinergic neurons as well as GABAergic neurons. Injection of 192IgG-saporin decreased the numbers of $\mathrm{BF}$ cholinergic neurons and GABAergic parvalbumin neurons by 69 and 11\%, respectively (Kaur et al, 2008), suggesting that neurotoxic lesion of cholinergic neurons also affected GABAergic neurons. Therefore, to clarify the roles of cholinergic neurons in the sleep-wake cycle, new approaches that can cell-selectively manipulate $\mathrm{BF}$ cholinergic neurons are needed.

Optogenetics is a novel tool that can be used to activate BF cholinergic neuron cell-specifically. Using ChAT-ChR2EYFP transgenic mice generated by a bacterial artificial chromosome transgenic approach, Han et al (2014) determined that activation of BF cholinergic neurons is sufficient to elicit an immediate transition from NREM sleep to wakefulness. In the present study, we found that selective activation of BF cholinergic neurons by DREADD increased wakefulness transiently and slightly, but decreased EEG delta power markedly and lastingly, inducing low-delta NREM sleep in mice. This inconsistency between the result of optogenetics and pharmacogenetics might result from different cellar mechanisms through which these techniques manipulate neuron activity (Farrell and Roth, 2013). Optogenetics utilizes light to activate ion channels and modulates the ion conductance of neuronal membranes transiently, while pharmacogenetics modulates G-proteinmediated signaling which can manipulate neurons activity for a longer time (Sasaki et al, 2011), allowing us to investigate the chronic and stable effects of manipulating $\mathrm{BF}$ cholinergic neurons.

The mice used to specifically activate BF cholinergic neurons in our and Han et al's studies are quite different too. Han et al (2014) used ChAT-ChR2-EYFP mice; it has been reported that these mice express ChR2 in cholinergic neurons of the brainstem laterodorsal tegmental nucleus (LDT) and pedunculopontine tegmental nucleus (PPT), as well as cholinergic neurons of the $\mathrm{BF}$ (Zhao et al, 2011). The cholinergic LDT/PPT neuron fibers, projecting toward the $\mathrm{BF}$, were in the range of the laser illumination and could have been activated when the BF was illuminated (Irmak and de Lecea, 2014; Losier and Semba, 1993). It has been reported that the cholinergic agonist carbachol strongly excited cortically projecting $\mathrm{BF}$ GABAergic neurons and increased their firing rate via muscarinic ACh receptors 1 and 3 (Yang et al, 2014). It is possible that the transition from NREM sleep to waking during light stimulation could result from the activation of cholinergic terminals from LDT/PPT neurons and neighboring GABAergic neurons. By contrast, we used ChAT-IRES-Cre mice and delivered viral vector via microinjection technology, ensuring that only the fibers of cholinergic neurons in the $\mathrm{BF}$, but not LDT/PPT, were activated after CNO injection. Therefore, the DREADD method, we applied in this study, can illustrate the exact role of $\mathrm{BF}$ cholinergic neurons in sleep-wake regulation.

The reduction of EEG delta power after activating $\mathrm{BF}$ cholinergic neurons may be attributed to the release of ACh in the cortex from terminals of these neurons. It has been shown that ACh increases the excitability of cortical pyramidal cells and promotes tonic firing by closing $\mathrm{K}^{+}$channels through muscarinic ACh receptors (Metherate et al, 1992). We mapped the projection of $\mathrm{MCPO} / \mathrm{SI} / \mathrm{HDB}$ cholinergic neurons and found that they project to the M2 and $\mathrm{Cg}$ abundantly. Moreover, we found that activation of BF cholinergic neurons by DREADD increased the expression of c-Fos in M2 and Cg, with a significant decrease of EEG delta power. These results suggest that BF cholinergic neurons decrease EEG delta activity through their innervations to the M2 and $\mathrm{Cg}$.

We also found that $\mathrm{BF}$ cholinergic neurons sparsely projected to the VLPO, an important structure responsible for behavioral sleep. Neurons of the VLPO increase firing rates (Szymusiak et al, 1998) and c-Fos expression (Scammell et al, 2000) during NREM sleep. The decrease of c-Fos expression in the VLPO after the activation of BF cholinergic neuron by DREADD is consistent with the previous findings that VLPO promoting sleep neurons were inhibited by cholinergic agonist in vitro (Gallopin et al, 2000), which may 
decrease the amount and depth of NREM sleep, as shown in our findings.

The TMN is a structure closely related to behavioral wakefulness with a significant increase of c-Fos expression during the waking state ( $\mathrm{Qu}$ et al, 2012; Scammell et al, 2001). We found that BF cholinergic neurons did not innervate the TMN and the activation of BF cholinergic neurons did not increase c-Fos expression in this region, supporting our findings that activation of $\mathrm{BF}$ cholinergic neurons results in a mild increase in wakefulness. These findings indicate that $\mathrm{BF}$ cholinergic neurons only play a minor role in promoting behavioral wakefulness, consistent with the proposition that sleep-wake state switching may be organized at the ascending reticular activating system of the brainstem (Pace-Schott and Hobson, 2002) and that BF cholinergic neurons serve as an relay from this brainstem system to the cerebral cortex.

EEG alpha activity is considered to be generated by thalamocortical neurons which are inhibited by GABAergic thalamic reticular nucleus (TRN) neurons (Steriade et al, 1987; von Krosigk et al, 1993). TRN has been shown to receive cholinergic innervation from the BF (Hallanger et al, 1987). Previous studies showed that ACh release evoked by electric stimulation (Sun et al, 2013) or optogenetic activation of cholinergic afferents in TRN (Pita-Almenar et al, 2014) elicited excitatory currents and triggered action potentials in TRN neurons. Therefore, activation of BF cholinergic neurons may increase the firing of GABAergic TRN neurons and lead to the inhibition of thalamocortical neurons and the decrease of alpha activity, as shown in the present study.

Beta activity is believed to be generated by cortical pyramidal neurons and relevant inhibitory feedback (Lacey et al, 2014). Jackson et al (2002) found that electrical stimulation of the pyramidal tract resets the phase of beta oscillations, and observed a subsequent suppression of corticospinal pyramidal neuron after the electrical stimulation. They proposed that the inhibitory feedback was the key mechanism generating beta activity, which imposed the oscillatory synchrony between pyramidal neurons during beta oscillation. It is possible that, after activating $\mathrm{BF}$ cholinergic neurons by pharmacogenetics, the released ACh increased the excitability of cortical pyramidal neurons but decreased the synchrony of these neurons. Therefore, the attenuation of the beta power spectrum in the present study may be attributed to the decrease of the synchrony between pyramidal neurons by $\mathrm{BF}$ cholinergic neurons. On the other hand, in addition to beta power spectrum, we also analyzed the beta power density after the injections of $\mathrm{CNO}$ at $0.1,0.3$, and $1.0 \mathrm{mg} / \mathrm{kg}$. Beta power density is calculated as beta power divided by the total power of the same epoch and indicates the relative change of beta power (Supplementary Method). We found that the beta power density was increased after the injection of $1.0 \mathrm{mg} / \mathrm{kg}$ $\mathrm{CNO}$, as compared with vehicle injection (Supplementary Figure S4). The increase of beta power density should be attributed to the significant decrease in delta power spectrum, indicating that $\mathrm{BF}$ cholinergic neurons primarily contribute to suppress delta activity.

Cholinergic terminals, arising from collaterals of $\mathrm{BF}$ cholinergic neurons, have been shown to terminate on neighboring BF GABAergic neurons (Yang et al, 2014;
Zaborszky and Duque, 2000), which innervate hippocampal interneurons and are suggested to be a crucial pacemaker for hippocampal theta activity (Freund and Antal, 1988; Yoder and Pang, 2005). Yang et al (2014) demonstrated that carbachol increased the firing rate of BF GABAergic neurons through muscarinic $\mathrm{M} 1$ and $\mathrm{M} 3$ receptors and optogenetic stimulation of BF cholinergic bodies/fibers caused a atropine-sensitive inward current in putative GABAergic neurons. They proposed that ACh may impact the H-currents of BF GABAergic neurons and modulate the burst or cluster firing of these neurons. It is reasonable to conjecture that the alteration of rhythmic firing of GABAergic neurons may contribute to the decrease of theta power after activating BF cholinergic neurons by pharmacogenetics, though the concrete mechanisms remain to be determined.

\section{CONCLUSION}

In summary, our results show that activation of $\mathrm{BF}$ cholinergic neurons markedly decreases EEG delta power and increases cortical c-Fos expression while inhibition of BF cholinergic neurons increases EEG delta power, indicating that the primary function of BF cholinergic neurons is to regulate EEG delta activity and promote cortical activity, rather than to induce behavioral wakefulness.

\section{FUNDING AND DISCLOSURE}

The authors have no proprietary interest in any materials or methods described within this article. This study was supported in part by grants-in-aid for scientific research from the National Basic Research Program of China (2015CB856401, to ZLH; 2011CB711000, to WMQ), the National Natural Science Foundation of China (31171010 and 31471064, to WMQ; 31421091, 31271164, 81420108015, and 31530035 , to ZLH), a key laboratory program of the Education Commission of Shanghai Municipality (ZDSYS14005, to ZLH), the Shanghai Committee of Science and Technology (14JC1400900, 13dz2260700, and 13140903100, to ZLH), and the World Premier International Research Center Initiative (WPI) from the Ministry of Education, Culture, Sports, Science, and Technology of Japan (to $\mathrm{YC}$ and $\mathrm{ML}$ ).

\section{REFERENCES}

Anaclet C, Ferrari L, Arrigoni E, Bass CE, Saper CB, Lu J et al (2014). The GABAergic parafacial zone is a medullary slow wave sleep-promoting center. Nat Neurosci 17: 1217-1224.

Arrigoni E, Chamberlin NL, Saper CB, McCarley RW (2006). Adenosine inhibits basal forebrain cholinergic and noncholinergic neurons in vitro. Neuroscience 140: 403-413.

Berntson GG, Shafi R, Sarter M (2002). Specific contributions of the basal forebrain corticopetal cholinergic system to electroencephalographic activity and sleep/waking behaviour. Eur J Neurosci 16: 2453-2461.

Blanco-Centurion C, Gerashchenko D, Shiromani PJ (2007). Effects of saporin-induced lesions of three arousal populations on daily levels of sleep and wake. J Neurosci 27: 14041-14048. 
Blanco-Centurion CA, Shiromani A, Winston E, Shiromani PJ (2006). Effects of hypocretin-1 in 192-IgG-saporin-lesioned rats. Eur J Neurosci 24: 2084-2088.

Cape EG, Jones BE (2000a). Effects of glutamate agonist versus procaine microinjections into the basal forebrain cholinergic cell area upon gamma and theta EEG activity and sleep-wake state. Eur J Neurosci 12: 2166-2184.

Cape EG, Manns ID, Alonso A, Beaudet A, Jones BE (2000b). Neurotensin-induced bursting of cholinergic basal forebrain neurons promotes gamma and theta cortical activity together with waking and paradoxical sleep. J Neurosci 20: 8452-8461.

Castillo-Ruiz A, Nixon JP, Smale L, Nunez AA (2010). Neural activation in arousal and reward areas of the brain in day-active and night-active grass rats. Neuroscience 165: 337-349.

Davis CJ, Clinton JM, Jewett KA, Zielinski MR, Krueger JM (2011). Delta wave power: an independent sleep phenotype or epiphenomenon? J Clin Sleep Med 7: S16-S18.

Farrell MS, Roth BL (2013). Pharmacosynthetics: reimagining the pharmacogenetic approach. Brain Res 1511: 6-20.

Freund TF, Antal M (1988). GABA-containing neurons in the septum control inhibitory interneurons in the hippocampus. Nature 336: 170-173.

Fuller PM, Sherman D, Pedersen NP, Saper CB, Lu J (2011). Reassessment of the structural basis of the ascending arousal system. J Comp Neurol 519: 933-956.

Gallopin T, Fort P, Eggermann E, Cauli B, Luppi PH, Rossier J et al (2000). Identification of sleep-promoting neurons in vitro. Nature 404: 992-995.

Gritti I, Henny P, Galloni F, Mainville L, Mariotti M, Jones BE (2006). Stereological estimates of the basal forebrain cell population in the rat, including neurons containing choline acetyltransferase, glutamic acid decarboxylase or phosphateactivated glutaminase and colocalizing vesicular glutamate transporters. Neuroscience 143: 1051-1064.

Hallanger AE, Levey AI, Lee HJ, Rye DB, Wainer BH (1987). The origins of cholinergic and other subcortical afferents to the thalamus in the rat. J Comp Neurol 262: 105-124.

Han Y, Shi YF, Xi W, Zhou R, Tan ZB, Wang H et al (2014). Selective activation of cholinergic basal forebrain neurons induces immediate sleep-wake transitions. Curr Biol 24: 693-698.

Hassani OK, Lee MG, Henny P, Jones BE (2009). Discharge profiles of identified GABAergic in comparison to cholinergic and putative glutamatergic basal forebrain neurons across the sleepwake cycle. J Neurosci 29: 11828-11840.

Huang ZL, Qu WM, Eguchi N, Chen JF, Schwarzschild MA, Fredholm BB et al (2005). Adenosine A2A, but not A1, receptors mediate the arousal effect of caffeine. Nat Neurosci 8: 858-859.

Irmak SO, de Lecea L (2014). Basal forebrain cholinergic modulation of sleep transitions. Sleep 37: 1941-1951.

Jackson A, Spinks RL, Freeman TC, Wolpert DM, Lemon RN (2002). Rhythm generation in monkey motor cortex explored using pyramidal tract stimulation. J Physiol 541: 685-699.

Jones BE (2004). Activity, modulation and role of basal forebrain cholinergic neurons innervating the cerebral cortex. Prog Brain Res. 145: 157-169.

Jones BE (2005). From waking to sleeping: neuronal and chemical substrates. Trends Pharmacol Sci 26: 578-586.

Kaur S, Junek A, Black MA, Semba K (2008). Effects of ibotenate and 192IgG-saporin lesions of the nucleus basalis magnocellularis/substantia innominata on spontaneous sleep and wake states and on recovery sleep after sleep deprivation in rats. $J$ Neurosci 28: 491-504.

Lacey MG, Gooding-Williams G, Prokic EJ, Yamawaki N, Hall SD, Stanford IM et al (2014). Spike firing and IPSPs in layer V pyramidal neurons during beta oscillations in rat primary motor cortex (M1) in vitro. PLoS One 9: e85109.

Lazarus M, Shen HY, Cherasse Y, Qu WM, Huang ZL, Bass CE et al (2011). Arousal effect of caffeine depends on adenosine A2A receptors in the shell of the nucleus accumbens. J Neurosci 31: 10067-10075.

Losier BJ, Semba K (1993). Dual projections of single cholinergic and aminergic brainstem neurons to the thalamus and basal forebrain in the rat. Brain Res 604: 41-52.

Manfridi A, Brambilla D, Mancia M (1999). Stimulation of NMDA and AMPA receptors in the rat nucleus basalis of Meynert affects sleep. Am J Physiol 277: R1488-R1492.

Metherate R, Cox CL, Ashe JH (1992). Cellular bases of neocortical activation: modulation of neural oscillations by the nucleus basalis and endogenous acetylcholine. J Neurosci 12: 4701-4711.

Pace-Schott EF, Hobson JA (2002). The neurobiology of sleep: genetics, cellular physiology and subcortical networks. Nat Rev Neurosci 3: 591-605.

Paxinos GT, Franklin K (2001). The Mouse Brain in Stereotaxic Coordinates, 2nd edn. Academic Press: San Diego.

Pita-Almenar JD, Yu D, Lu HC, Beierlein M (2014). Mechanisms underlying desynchronization of cholinergic-evoked thalamic network activity. J Neurosci 34: 14463-14474.

Portas CM, Thakkar M, Rainnie DG, Greene RW, McCarley RW (1997). Role of adenosine in behavioral state modulation: a microdialysis study in the freely moving cat. Neuroscience 79: 225-235.

Qu WM, Huang ZL, Xu XH, Matsumoto N, Urade Y (2008). Dopaminergic D1 and D2 receptors are essential for the arousal effect of modafinil. J Neurosci 28: 8462-8469.

Qu WM, Xu XH, Yan MM, Wang YQ, Urade Y, Huang ZL (2010). Essential role of dopamine D2 receptor in the maintenance of wakefulness, but not in homeostatic regulation of sleep, in mice. J Neurosci 30: 4382-4389.

Qu WM, Yue XF, Sun Y, Fan K, Chen CR, Hou YP et al (2012). Honokiol promotes non-rapid eye movement sleep via the benzodiazepine site of the GABA(A) receptor in mice. $\mathrm{Br} J$ Pharmacol 167: 587-598.

Rossi J, Balthasar N, Olson D, Scott M, Berglund E, Lee CE et al (2011). Melanocortin-4 receptors expressed by cholinergic neurons regulate energy balance and glucose homeostasis. Cell Metab 13: 195-204.

Sasaki K, Suzuki M, Mieda M, Tsujino N, Roth B, Sakurai T (2011). Pharmacogenetic modulation of orexin neurons alters sleep/ wakefulness states in mice. PLoS One 6: e20360.

Scammell TE, Estabrooke IV, McCarthy MT, Chemelli RM, Yanagisawa M, Miller MS et al (2000). Hypothalamic arousal regions are activated during modafinil-induced wakefulness. J Neurosci 20: 8620-8628.

Scammell TE, Gerashchenko DY, Mochizuki T, McCarthy MT, Estabrooke IV, Sears CA et al (2001). An adenosine A2a agonist increases sleep and induces Fos in ventrolateral preoptic neurons. Neuroscience 107: 653-663.

Semba K (2000). Multiple output pathways of the basal forebrain: organization, chemical heterogeneity, and roles in vigilance. Behav Brain Res 115: 117-141.

Steriade M, Domich L, Oakson G, Deschenes M (1987). The deafferented reticular thalamic nucleus generates spindle rhythmicity. J Neurophysiol 57: 260-273.

Sun YG, Pita-Almenar JD, Wu CS, Renger JJ, Uebele VN, Lu HC et al (2013). Biphasic cholinergic synaptic transmission controls action potential activity in thalamic reticular nucleus neurons. J Neurosci 33: 2048-2059.

Szymusiak R, Alam N, Steininger TL, McGinty D (1998). Sleepwaking discharge patterns of ventrolateral preoptic/anterior hypothalamic neurons in rats. Brain Res 803: 178-188.

Takahashi K, Lin JS, Sakai K (2009). Characterization and mapping of sleep-waking specific neurons in the basal forebrain and preoptic hypothalamus in mice. Neuroscience 161: 269-292.

von Krosigk M, Bal T, McCormick DA (1993). Cellular mechanisms of a synchronized oscillation in the thalamus. Science 261: $361-364$ 
Yang C, McKenna JT, Zant JC, Winston S, Basheer R, Brown RE (2014). Cholinergic neurons excite cortically projecting basal forebrain GABAergic neurons. J Neurosci 34: 2832-2844.

Yoder RM, Pang KC (2005). Involvement of GABAergic and cholinergic medial septal neurons in hippocampal theta rhythm. Hippocampus 15: 381-392.
Zaborszky L, Duque A (2000). Local synaptic connections of basal forebrain neurons. Behav Brain Res 115: 143-158.

Zhao S, Ting JT, Atallah HE, Qiu L, Tan J, Gloss B et al (2011). Cell type-specific channelrhodopsin-2 transgenic mice for optogenetic dissection of neural circuitry function. Nat Methods 8: $745-752$.

Supplementary Information accompanies the paper on the Neuropsychopharmacology website (http://www.nature.com/npp) 